\title{
Complete Genome Sequence of GD487, a High-Virulence Strain of Human-Associated ST398 Methicillin-Susceptible Staphylococcus aureus
}

\author{
Jo-Ann McClure, ${ }^{a}$ Steven M. Shideler, ${ }^{c}$ (i) Kunyan Zhang ${ }^{a, b, c, d, e}$ \\ aCentre for Antimicrobial Resistance, Alberta Health Services/Alberta Public Laboratories/University of Calgary, Calgary, Alberta, Canada \\ bDepartment of Pathology \& Laboratory Medicine, University of Calgary, Calgary, Alberta, Canada \\ 'Department of Microbiology, Immunology and Infectious Diseases, University of Calgary, Calgary, Alberta, Canada \\ 'Department of Medicine, University of Calgary, Calgary, Alberta, Canada \\ eThe Calvin, Phoebe and Joan Snyder Institute for Chronic Diseases, University of Calgary, Calgary, Alberta, Canada
}

ABSTRACT Multilocus sequence type 398 (ST398) methicillin-susceptible Staphylococcus aureus (MSSA) has been shown to have augmented pathogenicity in humans. However, it has not been determined whether all ST398 strains are equally virulent. We present here the genome sequence of a high-virulence ST398 MSSA strain, GD487, to explore potential insights into ST398 virulence.

taphylococcus aureus multilocus sequence type 398 (ST398) was first reported as an animal pathogen affecting livestock and companion animals but was soon found associated with human diseases, even in the absence of animal contact (1-17). In humans, ST398 methicillin-susceptible S. aureus (MSSA) strains are commonly isolated and have been recognized as the causative agents in a number of severe infections in young healthy people, suggesting that MSSA strains have augmented pathogenicity in humans $(2,8,18)$. Reports investigating the virulence of ST398 have focused on genetic factors of the group as a whole $(10,17,19)$, with no specifics as to whether there are virulence differences within the lineage. The Caenorhabditis elegans infection model has proven to be a robust model for investigating $S$. aureus virulence $(20,21)$. We compared the virulence of ST398 strains in our collection using the model. Preliminary analysis found three lineages with high, moderate, or low virulence, with mean nematode killing rates of $90 \%, 67 \%$, and $44 \%$, respectively. Whole-genome sequencing was done to determine if there were specific factors present that could contribute to the noted levels of virulence. In separate reports, we presented the genomic sequences of a moderate-virulence (GD1108) and a low-virulence (GD1696) strain. Here, we present the complete chromosomal sequence of high-virulence strain GD487 to explore potential insights into ST398 sublineage virulence.

Strain GD487 was isolated from a school child from a prevalence survey in 2011 in Guangzhou, People's Republic of China. Genomic DNA was isolated using phenolchloroform extraction from a $37^{\circ} \mathrm{C}$ overnight culture of a single colony in brain heart infusion (BHI) broth. PacBio library preparation and DNA sequencing were performed at the Genome Quebec Innovation Centre in Montreal, Canada. A sheared large-insert library was generated using Covaris g-Tubes and the SMRTbell template prep kit 1.0 and then sequenced with PacBio RS II sequencing technology using one singlemolecule real-time (SMRT) cell. Illumina library preparation and sequencing were performed at the Nicole Perkins Microbial Communities Core Laboratory at University of Calgary in Canada. The library was prepared using the Nextera XT library preparation kit using standard conditions, and then 600-cycle MiSeq v3 sequencing was done. Adapters were trimmed, and sequences with a quality score of $<20$ were removed from
Citation McClure J-A, Shideler SM, Zhang K 2019. Complete genome sequence of GD487, a high-virulence strain of human-associated ST398 methicillin-susceptible Staphylococcus aureus. Microbiol Resour Announc 8:e00686-19. https://doi.org/10.1128/MRA.00686-19. Editor Frank J. Stewart, Georgia Institute of Technology

Copyright $\odot 2019$ McClure et al. This is an open-access article distributed under the terms of the Creative Commons Attribution 4.0 International license.

Address correspondence to Kunyan Zhang, kzhang@ucalgary.ca.

Received 10 June 2019

Accepted 18 June 2019

Published 3 July 2019 
the raw Illumina reads with Cutadapt v1.15, and sequence quality was assessed with FastQC v0.11.5 (http://www.bioinformatics.babraham.ac.uk/projects/fastqc) (22). Hybrid genome assembly using the trimmed Illumina reads and filtered PacBio subreads (prepared by Genome Quebec) was done using the Unicycler v0.4.7 pipeline (SPAdes v3.13.0, minimap, Racon v1.3.2, and Pilon v.1.23), with GC content determined using QUAST v4.4 (23-28). Gene annotation was done using the NCBI Prokaryotic Genome Annotation Pipeline using the best-placed reference protein set (GeneMarkS-2+v4.8) (29). Default settings were used for each program.

Two contigs resulted from hybrid assembly of the GD487 reads, the chromosome and a plasmid (11, $006 \mathrm{bp})$. PacBio sequencing produced 98,884 raw reads covering $1,226,233,312$ sequenced bases, with an average read length of $12,400 \mathrm{bp}$. Illumina sequencing produced 383,925 reads, with an average read length of $213 \mathrm{bp}$ for R1 and $193 \mathrm{bp}$ for R2. The estimated genome coverages were $395 \times$ and $30 \times$ for PacBio and Illumina reads, respectively, with a GC content of $32.96 \%$ for the assembled product. The resulting chromosome was 2,758,447 bp long, with 2,787 genes identified, of which 2,709 were coding sequences (CDS), 78 were RNA genes, and 87 were pseudogenes.

Data availability. The chromosomal genome sequence was deposited at GenBank under the accession number CP040229 and SRA accession numbers SRX5914559 (Illumina) and SRX5914560 (PacBio).

\section{ACKNOWLEDGMENTS}

This work was supported in part by operation grants (ARE-147623 and ARF-151557) from the Canadian Institutes of Health Research, Canada, and in part by an operating fund from the Centre for Antimicrobial Resistance (CAR), Alberta Health Services, Alberta, Canada.

\section{REFERENCES}

1. Bradley A. 2002. Bovine mastitis: an evolving disease. Vet J 164:116-128. https://doi.org/10.1053/tvjl.2002.0724.

2. Brunel AS, Banuls AL, Marchandin H, Bouzinbi N, Morquin D, Jumas-Bilak E, Corne P. 2014. Methicillin-sensitive Staphylococcus aureus CC398 in intensive care unit, France. Emerg Infect Dis 20:1511-1515. https://doi .org/10.3201/eid2009.130225.

3. Cuny C, Friedrich A, Kozytska S, Layer F, Nubel U, Ohlsen K, Strommenger B, Walther B, Wieler L, Witte W. 2010. Emergence of methicillin-resistant Staphylococcus aureus (MRSA) in different animal species. Int J Med Microbiol 300:109-117. https://doi.org/10.1016/j.ijmm.2009.11.002.

4. Dorado-Garcia A, Bos ME, Graveland H, Van Cleef BA, Verstappen KM, Kluytmans JA, Wagenaar JA, Heederik DJ. 2013. Risk factors for persistence of livestock-associated MRSA and environmental exposure in veal calf farmers and their family members: an observational longitudinal study. BMJ Open 3:e003272. https://doi.org/10.1136/bmjopen -2013-003272.

5. Graveland H, Duim B, van Duijkeren E, Heederik D, Wagenaar JA. 2011. Livestock-associated methicillin-resistant Staphylococcus aureus in animals and humans. Int J Med Microbiol 301:630-634. https://doi.org/10 .1016/j.ijmm.2011.09.004.

6. McNamee PT, Smyth JA. 2000. Bacterial chondronecrosis with osteomyelitis ("femoral head necrosis") of broiler chickens: a review. Avian Pathol 29:253-270. https://doi.org/10.1080/03079450050118386.

7. Menzies PI, Ramanoon SZ. 2001. Mastitis of sheep and goats. Vet Clin North Am Food Anim Pract 17:333-358, vii. https://doi.org/10.1016/S0749 $-0720(15) 30032-3$.

8. Rasigade JP, Laurent F, Hubert P, Vandenesch F, Etienne J. 2010. Lethal necrotizing pneumonia caused by an ST398 Staphylococcus aureus strain. Emerg Infect Dis 16:1330. https://doi.org/10.3201/eid1608.100317.

9. Springer B, Orendi U, Much P, Hoger G, Ruppitsch W, Krziwanek K, Metz-Gercek S, Mittermayer H. 2009. Methicillin-resistant Staphylococcus aureus: a new zoonotic agent? Wien Klin Wochenschr 121:86-90. https:// doi.org/10.1007/s00508-008-1126-y.

10. Uhlemann AC, Dumortier C, Hafer C, Taylor BS, Sanchez J, RodriguezTaveras C, Leon P, Rojas R, Olive C, Lowy FD. 2012. Molecular characterization of Staphylococcus aureus from outpatients in the Caribbean reveals the presence of pandemic clones. Eur J Clin Microbiol Infect Dis 31:505-511. https://doi.org/10.1007/s10096-011-1339-2.

11. Uhlemann AC, Hafer C, Miko BA, Sowash MG, Sullivan SB, Shu Q, Lowy FD. 2013. Emergence of sequence type 398 as a community- and healthcare-associated methicillin-susceptible Staphylococcus aureus in northern Manhattan. Clin Infect Dis 57:700-703. https://doi.org/10.1093/ cid/cit375.

12. van Belkum A, Melles DC, Peeters JK, van Leeuwen WB, van Duijkeren $E$, Huijsdens XW, Spalburg E, de Neeling AJ, Verbrugh HA, Dutch Working Party on Surveillance Research of MRSA (SOM). 2008. Methicillinresistant and -susceptible Staphylococcus aureus sequence type 398 in pigs and humans. Emerg Infect Dis 14:479-483. https://doi.org/10.3201/ eid1403.070760.

13. van Duijkeren $E$, Moleman $M$, Sloet van Oldruitenborgh-Oosterbaan $M M$, Multem J, Troelstra A, Fluit AC, van Wamel WJ, Houwers DJ, de Neeling AJ, Wagenaar JA. 2010. Methicillin-resistant Staphylococcus aureus in horses and horse personnel: an investigation of several outbreaks. Vet Microbiol 141:96-102. https://doi.org/10.1016/j.vetmic.2009.08.009.

14. Voss A, Loeffen F, Bakker J, Klaassen C, Wulf M. 2005. Methicillin-resistant Staphylococcus aureus in pig farming. Emerg Infect Dis 11:1965-1966. https://doi.org/10.3201/eid1112.050428.

15. Weese JS. 2010. Methicillin-resistant Staphylococcus aureus in animals. ILAR J 51:233-244. https://doi.org/10.1093/ilar.51.3.233.

16. Witte W, Strommenger B, Stanek C, Cuny C. 2007. Methicillin-resistant Staphylococcus aureus ST398 in humans and animals, Central Europe. Emerg Infect Dis 13:255-258. https://doi.org/10.3201/eid1302.060924.

17. Yu F, Chen Z, Liu C, Zhang X, Lin X, Chi S, Zhou T, Chen Z, Chen X. 2008. Prevalence of Staphylococcus aureus carrying Panton-Valentine leukocidin genes among isolates from hospitalised patients in China. Clin Microbiol Infect 14:381-384. https://doi.org/10.1111/j.1469-0691 .2007.01927.x.

18. Valentin-Domelier AS, Girard M, Bertrand X, Violette J, Francois P, Donnio PY, Talon D, Quentin R, Schrenzel J, van der Mee-Marquet N, Bloodstream Infection Study Group of the Reseau des Hygienistes du Centre (RHC). 2011. Methicillin-susceptible ST398 Staphylococcus aureus responsible for bloodstream infections: an emerging human-adapted subclone? PLoS One 6:e28369. https://doi.org/10.1371/journal.pone.0028369. 
19. Bonesso MF, Yeh AJ, Villaruz AE, Joo HS, McCausland J, Fortaleza CM Cavalcante RS, Sobrinho MT, Ronchi CF, Cheung GY, Cunha ML, Otto M. 2016. Key role of alpha-toxin in fatal pneumonia caused by Staphylococcus aureus sequence type 398. Am J Respir Crit Care Med 193: 217-220. https://doi.org/10.1164/rccm.201506-1225LE.

20. Wu K, Conly J, McClure JA, Elsayed S, Louie T, Zhang K. 2010. Caenorhabditis elegans as a host model for community-associated methicillinresistant Staphylococcus aureus. Clin Microbiol Infect 16:245-254. https:// doi.org/10.1111/j.1469-0691.2009.02765.x.

21. Wu K, Simor AE, Vearncombe M, McClure JA, Zhang K. 2012. A Caenorhabditis elegans host model correlates with invasive disease caused by Staphylococcus aureus recovered during an outbreak in neonatal intensive care. Can J Infect Dis Med Microbiol 23:130-134. https://doi.org/10 .1155/2012/543817.

22. Martin M. 2011. Cutadapt removes adapter sequences from highthroughput sequencing reads. EMBnet J 17:10-12. https://doi.org/10 .14806/ej.17.1.200.

23. Bankevich A, Nurk $S$, Antipov D, Gurevich AA, Dvorkin M, Kulikov AS, Lesin VM, Nikolenko SI, Pham S, Prjibelski AD, Pyshkin AV, Sirotkin AV, Vyahhi N, Tesler G, Alekseyev MA, Pevzner PA. 2012. SPAdes: a new genome assembly algorithm and its applications to single-cell sequencing. J Comput Biol 19:455-477. https://doi.org/10.1089/cmb.2012.0021.
24. Li H. 2016. Minimap and miniasm: fast mapping and de novo assembly for noisy long sequences. Bioinformatics 32:2103-2110. https://doi.org/ 10.1093/bioinformatics/btw152.

25. Vaser R, Sovic I, Nagarajan N, Sikic M. 2017. Fast and accurate de novo genome assembly from long uncorrected reads. Genome Res 27: 737-746. https://doi.org/10.1101/gr.214270.116.

26. Walker BJ, Abeel T, Shea T, Priest M, Abouelliel A, Sakthikumar S, Cuomo CA, Zeng Q, Wortman J, Young SK, Earl AM. 2014. Pilon: an integrated tool for comprehensive microbial variant detection and genome assembly improvement. PLoS One 9:e112963. https://doi.org/10.1371/journal .pone.0112963.

27. Wick RR, Judd LM, Gorrie CL, Holt KE. 2017. Unicycler: resolving bacterial genome assemblies from short and long sequencing reads. PLoS Comput Biol 13:e1005595. https://doi.org/10.1371/journal.pcbi.1005595.

28. Gurevich A, Saveliev V, Vyahhi N, Tesler G. 2013. QUAST: quality assessment tool for genome assemblies. Bioinformatics 29:1072-1075. https:// doi.org/10.1093/bioinformatics/btt086.

29. Tatusova T, DiCuccio M, Badretdin A, Chetvernin V, Nawrocki EP, Zaslavsky L, Lomsadze A, Pruitt KD, Borodovsky M, Ostell J. 2016. NCBI Prokaryotic Genome Annotation Pipeline. Nucleic Acids Res 44:6614-6624. https://doi .org/10.1093/nar/gkw569. 